\title{
Bridging the gap between micros and minis in the teaching laboratory
}

\author{
PHILIP F. SPELT \\ Wabash College, Crawfordsville, Indiana 47933
}

\begin{abstract}
This paper briefly considers the differences between minicomputers and microcomputers and the implications of these differences for data-generating simulations. The important difference is that between a timesharing and a single-user system. Finally, a hybrid model of survey research is presented. This model has some unusual features and is suitable for either type of computer.
\end{abstract}

The title of this paper contains terms that are no longer applicable in the world of computers. The computing size of "microcomputers" has become large enough in the past few years that as much memory and computing power are now available with an Apple II, for instance, as was available on the DEC PDP-11/20 10 or 12 years ago. Moreover, the PDP-11/70 has total memory, computing power, and ability to drive multiple terminals that, 10 or 15 years ago, were available only in the biggest main frames. Now, Radio Shack advertises a vest-pocket version of the TRS-80, with a full alphanumeric keyboard and a single line of liquid-crystal diode readout.

While this converging of the so-called "micros" and "minis" has generally reduced the previously existing, well-known differences between them, the differences have not been eliminated altogether. It is still true, for example, that when the CPU of an elaborate timesharing system "goes down," all stations are "gone" until the system can be brought back on-line again. Such is not the case, of course, with a battery of micro machines, networked or not. Moreover, a dedicated machine permits real-time data gathering (such as reaction time measures), which is much more difficult on a timesharing system. On the other hand, despite the increasing memory sizes among micros, and despite their dedication to a single user, the old problems of size and speed still remain for the majority of the popular (and lower priced) units. For example, most popular micros are 8-bit machines that can use $64 \mathrm{~K}$ of memory by adopting a two-word memory addressing system, a tactic that consumes memory and slows down processing. There are also other aspects of the microcomputer configuration that slow processing. An example of the relative computing speeds is provided by a "heapsort" program that takes several orders of magnitude longer to sort on an Apple II than on a PDP-11/70. A major limitation for microcomputers is the size of the program or data base that can be accessed. Even in the case of a network of microcomputers, it is not possible presently to have multiple users accessing the same data file on the network disk. This proves to be a major handicap in running the standard EXPER SIM models on a micro network. One can, of course, make multiple copies on flexible diskettes, but this has drawbacks, including the speed of access on the diskettes. Nevertheless, improvements are being made, and when true networking arrives (as in ARPANET and TELENET), these size problems may disappear.

Therefore, when we talk of "bridging the gap" between micros and minis, we must do so at the conceptual, programming level, not at the level of machinery. In fact, the meaningful distinction is that between a timesharing and a single-user system, or personal computer. In this paper, I would like to illustrate some of the major differences between these two configurations as they apply to simulations designed for teaching research design and statistics.

\section{TIMESHARING AND SINGLE-USER SYSTEMS}

Figure 1 presents a generalized picture of an EXPER SIM-type simulation package. Such a package consists of a set of programs and a data file. In Figure 1, the square and trapezoidal elements are programs and the circle is the data file. The package consists of a set of programs

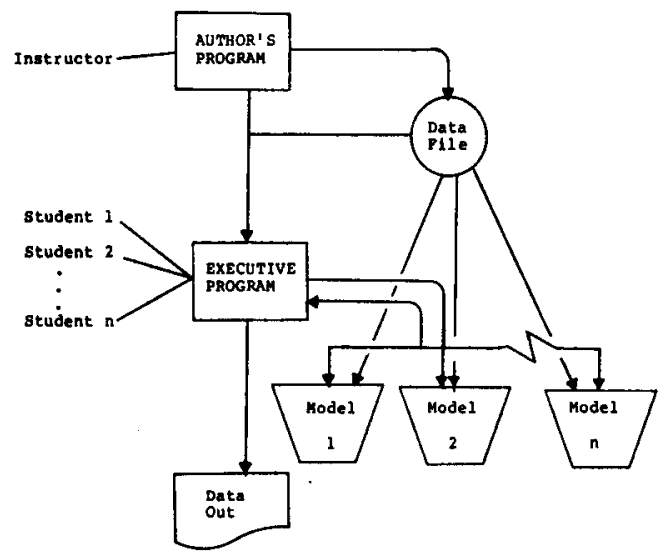

Figure 1. Typical timesharing simulation system, EXPER SIM type. 
that comprises the models and an executive program that intervenes between the student users and the various parameters to drive each of the models, which access the appropriate parts of the data file whenever they are run. These parameters are placed in a data file to facilitate their accessibility by both the executive and the model programs, by multiple users running either the same or different models.

The advantages of this system have been fully explored elsewhere (Main, 1971; Thurmond \& Cromer, 1972; Stout, Note 1), so I will review only some of the more pertinent ones. As stated above, the use of an executive program standardizes the various models' $1 / 0$ formats for the students and also helps protect the models from the probing minds and fingers of inquisitive, selfappointed "computer jocks." Moreover, although most of the models are not written this way, it is theoretically possible to modify a particular model by changing only the master data file, rather than altering the program that contains the model, although I am not aware of this actually being done in practice.

On a single-user system, the $\mathrm{I} / \mathrm{O}$ routines are repeated in each copy of the program, but this is of no concern in terms of memory size, since each routine is in a different machine. In addition, there is no need for the data file, since the parameters can be incorporated directly into the only program in the "system." The system presented in Figure 2 is a hybrid that works on either timesharing or personal computers. The various geometric figures have the same significance here as in Figure 1. The major difference between these two configurations is that in the single-user case, the model program must contain all $\mathrm{I} / \mathrm{O}$ routines. To the student user, there is no noticeable difference between the two configurations. The significant addition to the system presented in Figure 2, from the perspective of the instructor, is the use of a model-modifying program that permits significant changes in the model without programming knowledge; the changes are handled by a dialogue between the user and the machine. Thus, the model parameters are removed from the model program, as in the timesharing configuration, so that they can be "permanently" modified by means of the modelmodifying program.

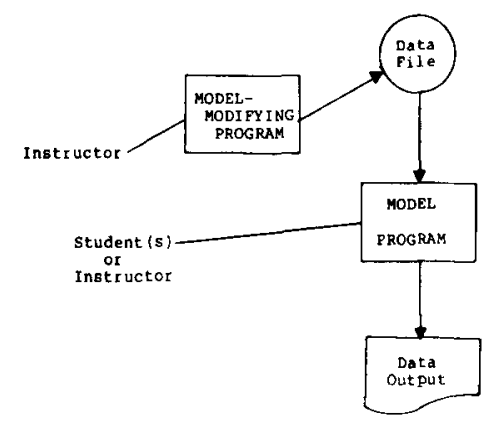

Figure 2. Hybrid simulation system suitable for either timesharing or single-user system.

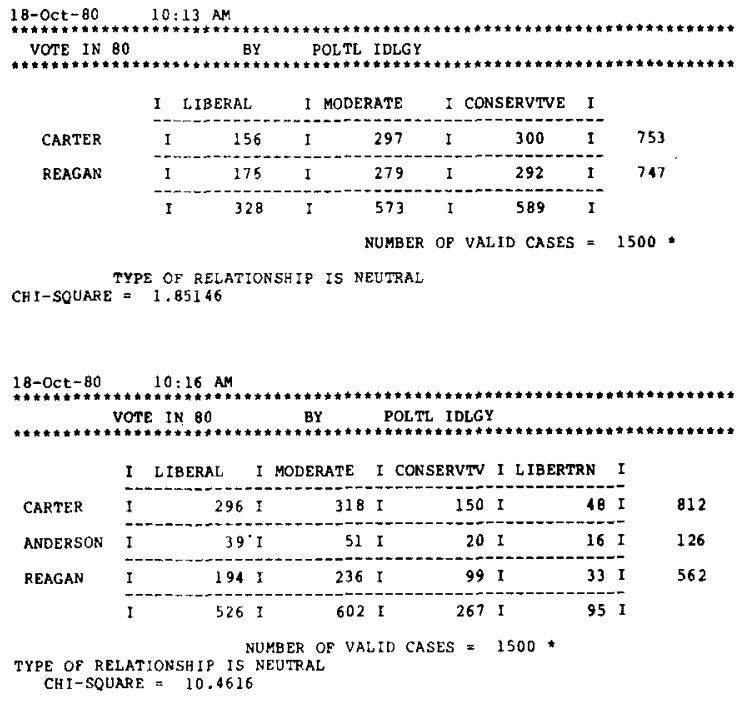

Figure 3. (a) Unaltered output matrix, (b) A 4 by 3 useraltered matrix from survey simulation.

An example of a model that fits the configuration of Figure 2 is one I am currently developing with C. Anthony Broh, a political scientist at Columbia University. This model is designed to teach students how to deal with data from survey research presented in tabular form. In its finished form, the model will come in a standard 2 by 3 table of political candidate (Democrat or Republican) by political party (Democratic, Independent, or Republican), an example of which is presented in Figure 3a. The cell percentages will be set to match those from the 1980 election, and when the student runs the model, she/he will receive a table with a format that matches that produced by the SPSS package. When running the model program, the student has a choice of a variety of variables to set, including the presence of missing data, the sample size, and the use of a control variable. In addition, the labels on the rows and columns, the size of the table, and the cell percentages can be altered if the student so chooses. These changes are made during each student's run and for that run only. Thus, the student can explore a variety of aspects of survey research data format and relationships without affecting other students' use of the basic model. Once that run of the model is over, the changes made by the user "go away," and the next time that model is run, it will be back to the basic configuration.

A more significant aspect of this hybrid system is represented by the model-modifying program, available only to the instructor. This program enables the instructor to "permanently" modify the basic configuration that the students see when they choose to run the simulation with the table configuration unaltered. With this program, the teacher can alter all labels, the overall table size, and the cell percentages. As indicated earlier, these changes are all done by means of an interactive dialogue between the program and the instructor. The changes are made to the model parameters in the data 
file, not to the model program itself. Thus, when the figures on the national voting pattern come from a new election, it will be a relatively trivial matter to update the cell percentages and, thus, keep the model current. Moreover, a social psychologist interested in survey research on, say, people's attitudes about belonging to a local country club can easily alter the model to simulate either real or hypothetical data from such a survey. Figure 3 shows two tables, one the current standard (Figure 3a) and the other (Figure 3b) my invention based on the 1980 presidential election (done well in advance).

Thus, the survey model could run on either a timesharing system or a personal machine. In the former case, there could be multiple student users at the point of the model program in Figure 2, whereas the latter case would, of course, have only a single user. Also, on a personal computer, the instructor can alter the model merely by loading the modification program and changing the parameters in the data file. The main advances incorporated in this model are the ease with which substantial changes, either temporary or per- manent, can be made in the basic model without any programming being required. These concepts can also be applied to other models, including those in the various EXPER SIM configurations. However, in order to allow existing models to be so altered, considerable reprogram. ming would be required, and I doubt that the effort would be justified.

\section{REFERENCE NOTE}

1. Stout, R. L. Programmer's guide to MESS, Versions 3-P, 3-SB, and 3-SC. Ann Arbor: University of Michigan, February 25, 1974.

\section{REFERENCES}

Main D. B., \& Head, S. Computer simulations in the elementary psychology laboratory. Proceedings of the Conference on Computers in Undergraduate Curricula, Dartmouth, N.H., 1971.

Thurmond, J. B., \& Cromer, A. O. Toward the optimal use of computer simulations in teaching scientific research strategy. Proceedings of the Conference on Computers in Undergraduate Curricula, Atlanta, Ga., 1972. 